\title{
The co-evolution of culturally inherited altruistic helping and cultural transmission under random group formation
}

\author{
Laurent Lehmann*, Marcus W. Feldman \\ Department of Biological Sciences, Stanford University, USA
}

\section{A R T I C L E I N F O}

\section{Article history:}

Received 16 October 2007

Available online 13 March 2008

\section{Keywords:}

Altruism

Relatedness

Group selection

Cultural transmission

Genetic transmission

Conformist transmission

\begin{abstract}
A B S T R A C T
Limited migration results in kin selective pressure on helping behaviors under a wide range of ecological, demographic and life-history situations. However, such genetically determined altruistic helping can evolve only when migration is not too strong and group size is not too large. Cultural inheritance of helping behaviors may allow altruistic helping to evolve in groups of larger size because cultural transmission has the potential to markedly decrease the variance within groups and augment the variance between groups. Here, we study the co-evolution of culturally inherited altruistic helping behaviors and two alternative cultural transmission rules for such behaviors. We find that conformist transmission, where individuals within groups tend to copy prevalent cultural variants (e.g., beliefs or values), has a strong adverse effect on the evolution of culturally inherited helping traits. This finding is at variance with the commonly held view that conformist transmission is a crucial factor favoring the evolution of altruistic helping in humans. By contrast, we find that under one-to-many transmission, where individuals within groups tend to copy a "leader" (or teacher), altruistic helping can evolve in groups of any size, although the cultural transmission rule itself hitchhikes rather weakly with a selected helping trait. Our results suggest that culturally determined helping behaviors are more likely to be driven by "leaders" than by popularity, but the emergence and stability of the cultural transmission rules themselves should be driven by some extrinsic factors.
\end{abstract}

Published by Elsevier Inc.

\section{Introduction}

Identifying all the conditions conducive to the emergence and stability of helping behaviors remains one of the challenges of behavioral ecology (Bshary and Bergmuller, 2007). When a population consists of groups that are of small size and migration is limited between groups, group members tend to be genetically related. This occurs because individuals within groups are more likely to have inherited (through the joint action of genetic drift and limited migration) identical alleles from a common ancestor than individuals sampled at random from the population. Such genetic relatedness between group members can account for the evolution of altruistic helping by kin selection under a wide range of biological scenarios in spatially subdivided populations (e.g., Hamilton (1971), Eshel (1972), Hamilton (1975), Aoki (1982), Rogers (1990), Taylor (1992), van Baalen and Rand (1998), Taylor and Irwin (2000), Le Galliard et al. (2003), Axelrod et al. (2004), Gardner and West (2006), Lehmann et al. (2006), Lehmann (2006), Jansen and van Baalen (2006), Ohtsuki et al. (2006), Grafen (2007a),

\footnotetext{
* Corresponding author.

E-mail addresses: lehmann@stanford.edu (L. Lehmann), marc@charles.stanford.edu (M.W. Feldman).
}

Grafen (2007b), Rousset and Roze (2007) and Lions and van Baalen (2008)). But genetic relatedness between interacting individuals decreases as group size increases. This occurs because the rate at which different individuals within groups inherit an allele from the same ancestor decreases as group size increases. Relatedness also decreases as the migration rate increases, because migration homogenizes the genetic composition of populations. With large group size or a high migration rate or both, the genetic variance within groups will be high and the variance between groups low (i.e., low relatedness), so that the evolution of genetically-inherited altruistic helping becomes unlikely.

Behaviors can also be inherited culturally. It has been suggested that the cultural inheritance of altruistic helping could be more favorable to its evolution in groups of large size than genetic inheritance (Boyd and Richerson, 1982; Fehr et al., 2002; Henrich, 2004; Richerson and Boyd, 2005; Boyd and Richerson, 2005). When a trait is inherited culturally, its transmission between the individuals in a population need not be strictly vertical but may also be oblique or horizontal (Cavalli-Sforza and Feldman, 1981). For instance, birds learn song dialects from neighboring individuals (Bonner, 1980), chimpanzees acquire the skills of "termiting" with tools by imitating group-mates (Goodall, 1966), and humans are able to learn from unrelated individuals present and from past generations (Cavalli-Sforza and Feldman, 1981; 
Lumsden and Wilson, 1981; Boyd and Richerson, 1985). With non-vertical cultural transmission comes the potential to pass on complex behavioral traits to biologically unrelated individuals. This may increase the coalescence rate of homologous cultural variants within groups, which, in turn, increases the cultural relatedness between group members relative to the relatedness under genetic transmission.

The distribution of cultural variants within and between groups depends on how individuals assimilate variants from other individuals in their group or the population (i.e., social learning). Each individual is characterized by a particular assimilation rule, which determines how he/she learns from other individuals. Assimilation rules can be divided into two non-mutually exclusive categories. First, an assimilation rule may depend on the frequency of cultural variants within a group. For instance, individuals may express preferences for common (conformist transmission) or to minority (contrariness) variants. Individuals may also have preferences for a partial consensus, thereby adopting variants in a manner to maintain a polymorphism in the group (Lumsden and Wilson, 1980, Figure 2). Conformist transmission, where individuals tend to adopt common variants and increase the frequency of these variants, has been proposed as the primary assimilation rule allowing high cultural relatedness to be maintained between group members (Henrich and Boyd, 2001; Henrich, 2004), and favoring the evolution of culturally-inherited altruistic helping in large groups (Boyd and Richerson, 1982; Richerson and Boyd, 2005; Boyd and Richerson, 2005).

Second, an assimilation rule may be independent of the frequency of cultural variants in the group or the population. In this case individuals assimilate cultural variants from other individuals, possibly according to the class or status of the exemplar individual (such as sex, age, wealth, prestige, etc.), so that one or several particular individuals may play a more important role than others in the transmission process.

Provided there is no statistical association between class and variants, cultural variants are neutral during cultural transmission. This situation can lead to strong cultural drift within groups (Cavalli-Sforza and Feldman, 1973, 1981), especially if the variance in cultural transmission is high. A high variance in cultural transmission results from individuals from different classes contributing very differently to the cultural transmission process (i.e., class structured populations or social hierarchies, CavalliSforza and Feldman (1981, pp. 315-339)). Suppose for instance that everybody in a group copies the cultural variant from a randomly chosen leader or teacher in the group with probability $\tau$, while with complementary probability $1-\tau$ the cultural variant of another individual sampled at random in the group is adopted (Cavalli-Sforza and Feldman, 1981, chapter 3.11). Here there are two classes of individuals: the leader and the other individuals. If groups are of large size and migration is frequent, the probability that two individuals bear the same cultural variant is approximatively $\tau^{2}$, which can be interpreted as the coefficient of cultural relatedness between these individuals, and is independent of group size. If $\tau$ is large, cultural relatedness can be very high in groups of any size and under any migration rate (Lehmann et al., 2007, in press).

Two questions concerning the evolution of culturally-inherited helping behaviors emerge from these considerations. First, what types of assimilation rules, frequency-dependent or frequencyindependent but class-based, are more likely to support both the emergence and stability of altruistic helping in groups of large size? Second, since the assimilation rules can lead to strong assortment between helpers, and helpers may concomitantly increase the fitness of individuals in their group, the fitness of individuals bearing assimilation rules leading to assortment of helpers may also increase. One might then wonder whether the genes determining the assimilation rules will actually coevolve with helping through hitchhiking? In this paper we address these questions by studying the co-evolution between culturally-inherited helping and two different assimilation rules in populations subject to random group formation and horizontal cultural transmission. We first ask whether helping can co-evolve with a one-to-many transmission rule, where individuals within groups tend to copy the cultural variants (e.g., beliefs or values) of a randomly chosen leader (or teacher). Second, we ask whether helping can co-evolve with conformist transmission. Here, we consider frequency-dependent assimilation rules according to which individuals comply with high frequency or low frequency behaviors in their group, and this allows us to cover the range from conformism to contrariness.

\section{Model}

\subsection{Life cycle}

Suppose the population consists of an infinite number of groups, each with $N$ adult individuals. We postulate that each individual is haploid and that there are two loci determining two distinct behaviors. The first locus controls the expression of a helping behavior that is inherited culturally; we call this the helping locus. The second locus determines the assimilation rule of the behavior at the helping locus. This modifier locus is envisioned here as being inherited genetically, although nothing in the following analysis prevents an interpretation in terms of cultural inheritance of this locus. We assume that two variants segregate at each locus. An individual bearing a helping variant (say $A)$ at the helping locus expresses a behavior that results in a fitness $\operatorname{cost} C_{\mathrm{A}}$ to him/herself but generates a fitness benefit $B$, which is shared among all group members except the actor (symbols are summarized in Table 1). By contrast, an individual bearing the second variant (say $a$ ) at the helping locus derives the benefit from expression of $A$ in others but pays no cost. An individual bearing the modifier variant $M$ at the modifier locus will retain or change, according to a horizontal transmission rule, its cultural variant at the helping locus inherited from its parent. We also assume that bearing variant $M$ can result in some fitness $\operatorname{cost} C_{\mathrm{M}}$. Individuals with variant $m$ at the modifier locus always retain the strategy at the helping locus that they inherited from their parents (i.e, strict vertical transmission), and suffer no fitness cost. In this twolocus two-variant setting, four pheno-genotypes segregate in the population ( $A M, A m, a M, a m)$.

The sequence of life cycle events is the following. (1) Adult individuals interact within groups. The survival probability of each individual then depends on its pheno-genotype and the pheno-genotypes of its neighbors. (2) Groups are dissolved and individuals then mate randomly in the population producing offspring that inherit the cultural variants from their parents, with recombination rate $r$ between the helping and the modifier loci. Adults then die. (3) Juveniles form new groups of constant size $N$. (4) Horizontal cultural transmission occurs according to the pheno-genotypes of individuals within groups.

We will investigate the effect of selection on two different forms of assimilation rule for the cultural transmission of helping behaviors. (i) Under one-to-many transmission, all individuals bearing variant $M$ in a group are assumed to copy the cultural variant at the helping locus from a randomly sampled individual (the leader or the teacher of the group). Since the leader can be any individual from the group, it bears the helping variant $A$ with probability equal to the frequency of $A$ in the group. Because all individuals bearing variant $M$ in the group adopt the cultural variant of the same leader, they will all have (after horizontal transmission) either pheno-genotype $A M$, if the leader 
Table 1

List of symbols

\begin{tabular}{|c|c|}
\hline Symbol & Definition \\
\hline$A$ & Helping variant. \\
\hline$a$ & Defector variant. \\
\hline$M$ & Modifier variant resulting in non-vertical cultural transmission. \\
\hline$m$ & Modifier variant resulting only in vertical cultural transmission. \\
\hline$C_{\mathrm{A}}$ & Fitness cost of expressing the helping variant $A$. \\
\hline$B$ & Fitness benefit generated from the expression of the helping variant $A$. \\
\hline$C_{\mathrm{M}}$ & Fitness cost of expressing the modifier variant $M$. \\
\hline$\alpha$ & $\begin{array}{l}\text { Parameter tuning the conformist effect from perfect contrariness }(\alpha \rightarrow-\infty) \text { to complete conformism }(\alpha \rightarrow \infty) \text {. If } \alpha=1 \text {, conformist transmission is } \\
\text { unbiased. }\end{array}$ \\
\hline$r$ & Recombination rate. \\
\hline$p_{\mathrm{A}}$ & Frequency of the helping variant $A$ after reproduction. \\
\hline$p_{M}$ & Frequency of the modifier variant $M$ after reproduction. \\
\hline$P_{j}$ & Frequency of pheno-genotype $j$ after reproduction. \\
\hline$X_{j}$ & Frequency of pheno-genotype $j$ after recombination. \\
\hline$N$ & Group size. \\
\hline$N_{j}$ & Number of individuals with pheno-genotype $j$ in a group after cultural transmission and just before reproduction. \\
\hline$N_{j}^{\star}$ & Number of individuals with pheno-genotype $j$ in a group just before cultural transmission. \\
\hline $\mathbf{N}$ & Vector giving the distribution of pheno-genotypes in a group after cultural transmission. \\
\hline $\operatorname{Pr}(\mathbf{N})$ & Probability distribution of pheno-genotypes in a group. \\
\hline$w_{i \mathbf{N}}$ & Fitness of individual $i$ in a group of type $\mathbf{N}$. \\
\hline $\bar{w}$ & Mean fitness in the population. \\
\hline
\end{tabular}

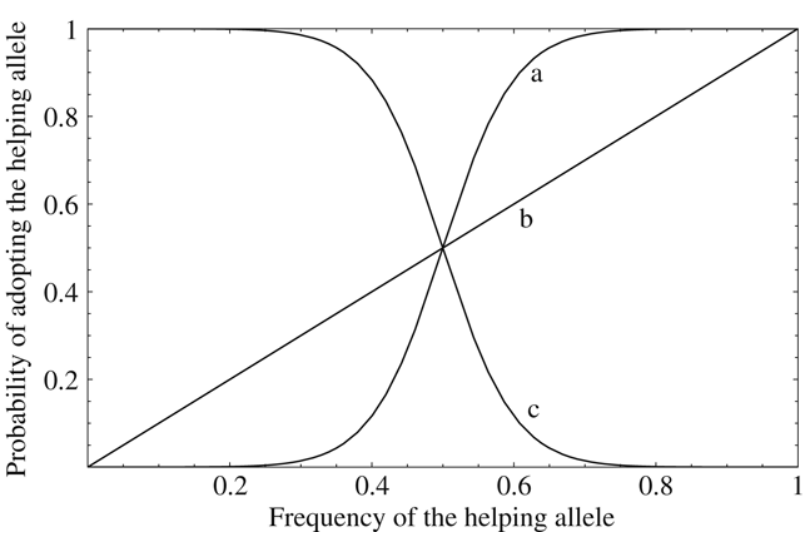

Fig. 1. Probability of adopting variant $A$ given by Eq. (1) and graphed as a function of the frequency of $A$ in a group. The sigmoidal curve (labeled a) corresponds to $\alpha=5$ and describes strong biased conformist transmission. The straight line (labeled $\mathrm{b}$ ) corresponds to $\alpha=1$ and shows the situation where the probability of adopting variant $A$ is equal to the frequency of $A$ in a group. In this case, conformist transmission is unbiased and variant $A$ is neutral during cultural transmission. The mirror image of the sigmoid curve (labeled c) corresponds to $\alpha=-5$ and describes strong contrariness where individuals prefer the less common variant in a group.

bears variant $A$, or they will all have pheno-genotype $a M$, if the leader bears variant $a$. (ii) Under conformist transmission, each individual bearing variant $M$ independently adopts one of the two cultural variants at the helping locus according to a conformist assimilation rule. We assume that a focal individual expressing variant $M$ adopts variant $A$ with probability

$u\left(x_{\mathrm{A}}\right)=\frac{x_{\mathrm{A}}^{\alpha}}{x_{\mathrm{A}}^{\alpha}+\left(1-x_{\mathrm{A}}\right)^{\alpha}}$,

where $x_{\mathrm{A}}$ is the frequency of variant $A$ in the focal individual's group and the parameter $\alpha$ allows us to tune the conformist effect from perfect conformism to complete contrariness (see Fig. 1 and Nakahashi (2007)). With negative values of the parameter $\alpha$ individuals are more willing to choose the less frequent cultural variant within their group (contrariness). When $\alpha=1$, individuals adopt variant $A$ with a probability equal to its frequency in the group, that is, conformist transmission is unbiased (Nakahashi, 2007), and variant $A$ is neutral in this case (i.e., no systematic change in frequency of variant $A$ occur during cultural transmission). When $\alpha>1$, conformist transmission is said to be biased (Boyd and Richerson, 2005).

\subsection{Variant dynamics}

The change in the average frequency $p_{\mathrm{K}}$ of variant $K(A$ or $M)$ in the population over one iteration of the life cycle can be written according to the Price equation (Price, 1970; Frank, 1998) as

$\bar{w} \Delta p_{\mathrm{K}}=\operatorname{Cov}\left[w_{i \mathbf{N}}, p_{\mathrm{K}(i \mathbf{N})}\right]+\mathrm{E}\left[w_{i \mathbf{N}} \Delta p_{\mathrm{K}(i \mathbf{N})}\right]$,

where $p_{\mathrm{K}(\mathrm{iN})}$ is the frequency ( 0 or 1 ) of variant $K$ after cultural transmission (and before reproduction) in individual $i$ in a group of type $\mathbf{N} \equiv\left(N_{\mathrm{AM}}, N_{\mathrm{Am}}, N_{\mathrm{aM}}\right)$, where $N_{j}$ is the number of individuals of pheno-genotype $j$ in a group (since $N_{\mathrm{AM}}+N_{\mathrm{Am}}+N_{\mathrm{aM}}+N_{\mathrm{am}}=N$, we have only three degrees of freedom and we can omit $N_{\mathrm{am}}$ from $\mathbf{N}$, for simplicity). Hence, $\mathbf{N}$ is a vector giving the number of individuals with each pheno-genotype in a group after cultural transmission, $w_{i \mathbf{N}}$ denotes the fitness of individual $i$ in a group of type $\mathbf{N}$, and $\bar{w}$ is the average fitness of individuals in the population. The covariance and the expectation in the Price equation are averages over all individuals within a group and all group types in the population. The first term in the Price equation represents the change in variant frequency due to selection while the second term represents the change in variant frequency due to other factors. In our model, these other factors are horizontal cultural transmission within groups at stage (4) of the life cycle.

From our assumptions, the fitness of individual $i$ in a group of type $\mathbf{N}$ is given by

$w_{i \mathbf{N}}=1-C_{\mathrm{A}} p_{\mathrm{A}(i \mathbf{N})}-C_{\mathrm{M}} p_{\mathrm{M}(i \mathbf{N})}+B p_{\mathrm{A}(-i \mathbf{N})}$,

where $p_{\mathrm{A}(-i \mathbf{N})}$ denotes the average frequency of variant $A$ in a group of type $\mathbf{N}$, but excluding individual $i$ from the average. Substituting Eq. (3) into Eq. (2), and noting that the change in frequency of variant $A$ due to horizontal cultural transmission is independent of the fitness of the pheno-genotypes, we find that the change in frequency $p_{\mathrm{A}}$ of the helping variant in the population can be written as

$\bar{w} \Delta p_{\mathrm{A}}=\left(-C_{\mathrm{A}}-C_{\mathrm{M}} R_{\mathrm{AM} \cdot \mathrm{A}}+B R_{\mathrm{A} / \mathrm{A}}\right) \operatorname{Var}\left[p_{\mathrm{A}(i \mathbf{N})}\right]+\bar{w} \mathrm{E}\left[\Delta p_{\mathrm{A}(\mathrm{iN})}\right]$,

where $\operatorname{Var}\left[p_{\mathrm{A}(\mathrm{N})}\right]$ is the variance of the frequency of variant $A$ among individuals across all groups in the population after cultural transmission,

$R_{\mathrm{AM} \cdot \mathrm{A}}=\frac{\operatorname{Cov}\left[p_{\mathrm{M}(\mathrm{iN})}, p_{\mathrm{A}(\mathrm{iN})}\right]}{\operatorname{Var}\left[p_{\mathrm{A}(\mathrm{iN})}\right]}$ 
is the regression of the variant at the modifier locus of a focal individual on the variant at the helping locus of that individual, and

$R_{\mathrm{A} / \mathrm{A}}=\frac{\operatorname{Cov}\left[p_{\mathrm{A}(-i \mathbf{N})}, p_{\mathrm{A}(\mathrm{iN})}\right]}{\operatorname{Var}\left[p_{\mathrm{A}(\mathrm{iN})}\right]}$

is the regression of the cultural variant at the helping locus of group-mates on the variant at the helping locus of a focal individual. Thus, $R_{\mathrm{A} / \mathrm{A}}$ can be viewed as a measure of cultural relatedness between interacting individuals (Allison, 1991). The term in parentheses in Eq. (4) is the gradient of selection on the helping variant $A$, and consists of three terms: the direct cost $C_{A}$ of bearing the helping variant $A$, the direct cost $C_{\mathrm{M}}$ of bearing the modifier variant $M$, which is weighted by the association between variants $A$ and $M$ within the same individual, and the indirect benefit $B$ stemming from other individuals in the group that carry $A$ and therefore offer help, which is weighted by the association of variant $A$ in two different individuals from the same group. The last term in Eq. (4) gives the change in the frequency of variant $A$ solely due to horizontal cultural transmission, which results from individuals within groups expressing the modifier variant $M$.

According to our assumptions, variant $M$ does not change in frequency during horizontal cultural transmission. From Eqs. (3) and (2), the change in frequency $p_{M}$ of the modifier variant $M$ can then be written as

$\bar{w} \Delta p_{\mathrm{M}}=\left(-C_{\mathrm{M}}-C_{\mathrm{A}} R_{\mathrm{AM} \cdot \mathrm{M}}+B R_{\mathrm{A} / \mathrm{M}}\right) \operatorname{Var}\left[p_{\mathrm{M}(i \mathbf{N})}\right]$,

where $\operatorname{Var}\left[p_{\mathrm{M}(\mathrm{iN})}\right]$ is the variance of the frequency of variant $M$ among individuals across all groups in the population after cultural transmission,

$R_{\mathrm{AM} \cdot \mathrm{M}}=\frac{\operatorname{Cov}\left[p_{\mathrm{A}(\mathrm{iN})}, p_{\mathrm{M}(\mathrm{iN})}\right]}{\operatorname{Var}\left[p_{\mathrm{M}(\mathrm{iN})}\right]}$

is the regression of the variant at the helping locus of a focal individual on the variant at the modifier locus of that individual, and

$R_{\mathrm{A} / \mathrm{M}}=\frac{\operatorname{Cov}\left[p_{\mathrm{A}(-i \mathbf{N})}, p_{\mathrm{M}(\mathbf{i N})}\right]}{\operatorname{Var}\left[p_{\mathrm{M}(i \mathbf{N})}\right]}$

is the regression of the genotype at the helping locus of groupmates on the genotype at the modifier locus of a focal individual. The gradient of selection on variant $M$ also depends on three terms: the direct $\operatorname{cost} C_{\mathrm{M}}$ to an individual bearing $M$, the direct $\operatorname{cost} C_{\mathrm{A}}$ to an individual bearing $A$, weighted by the association between variants $A$ and $M$ within the same individual, and the indirect benefit $B$ stemming from other individuals in the group expressing variant $A$, weighted by the association of variants $A$ and $M$ in two different individuals from the same group.

\subsection{Group type distribution}

In order to determine the evolutionary dynamics of the helping and modifier variants, we must evaluate the regressions of variant frequencies ( $R_{\mathrm{A} / \mathrm{A}}, R_{\mathrm{AM} \cdot \mathrm{A}}, R_{\mathrm{A} / \mathrm{M}}$ and $\left.R_{\mathrm{AM} \cdot \mathrm{M}}\right)$ appearing in Eqs. (4) and (7). To that end, we need the distribution $\operatorname{Pr}(\mathbf{N})$ of group types during the interaction stage (stage (1) of the life cycle), which occurs right after horizontal cultural transmission. This distribution can be expanded in terms of the distribution before cultural transmission according to

$\operatorname{Pr}(\mathbf{N})=\sum_{\mathbf{N}^{\star}} \operatorname{Pr}\left(\mathbf{N} \mid \mathbf{N}^{\star}\right) \operatorname{Pr}\left(\mathbf{N}^{\star}\right)$,

where $\operatorname{Pr}\left(\mathbf{N} \mid \mathbf{N}^{\star}\right)$ is the probability that a group of type $\mathbf{N}^{\star}$ before cultural transmission becomes a group of type $\mathbf{N}$ after cultural transmission, and $\operatorname{Pr}\left(\mathbf{N}^{\star}\right)$ is the distribution of group types just before cultural transmission. Different schemes of horizontal transmission will result in different group types even if group composition before cultural transmission is the same. This is because $\operatorname{Pr}\left(\mathbf{N} \mid \mathbf{N}^{\star}\right)$ depends on the assimilation rules expressed by the individuals in the population. In the Appendix, we present these cultural transition kernels for one-to-many and conformist transmissions (see Eqs. (A.1) and (A.2)). Finally, we need the distribution of group types $\operatorname{Pr}\left(\mathbf{N}^{\star}\right)$ just before cultural transmission. From our assumption of random group formation, this is given by the multinomial distribution

$$
\begin{aligned}
\operatorname{Pr}\left(\mathbf{N}^{\star}\right)= & \operatorname{Pr}\left(N_{\mathrm{AM}}^{\star}=h, N_{\mathrm{Am}}^{\star}=i, N_{\mathrm{aM}}^{\star}=j\right) \\
= & \frac{N !}{h ! i ! j !(N-h-i-j) !} \\
& \times X_{\mathrm{AM}}^{h} X_{\mathrm{Am}}^{i} X_{\mathrm{aM}}^{j}\left(1-X_{\mathrm{AM}}-X_{\mathrm{Am}}-X_{\mathrm{aM}}\right)^{N-h-i-j},
\end{aligned}
$$

where $X_{k}$ is the frequency of pheno-genotype $k$ in the population before group formation. Recombination between phenogenotypes entails that

$X_{\mathrm{AM}}=(1-r) P_{\mathrm{AM}}+r p_{\mathrm{A}} p_{\mathrm{M}}$

$X_{\mathrm{Am}}=(1-r) P_{\mathrm{Am}}+r p_{\mathrm{A}}\left(1-p_{\mathrm{M}}\right)$

$X_{\mathrm{aM}}=(1-r) P_{\mathrm{aM}}+r\left(1-p_{\mathrm{A}}\right) p_{\mathrm{M}}$,

where $P_{k}$ stands for the frequency of pheno-genotype $k$ in the population after reproduction but before recombination, while the frequencies $p_{\mathrm{A}}$ and $p_{\mathrm{M}}$ of variants $A$ and $M$ are not changed by recombination. When the modifier locus is envisioned to be inherited genetically (as is done here) it is probably best to consider free recombination $(r=1 / 2)$, because this corresponds to a situation where the cultural variant of an individual has an equal chance to have been inherited from its mother or from its father.

\subsection{Linkage disequilibrium}

The distribution of group types $\operatorname{Pr}(\mathbf{N})$ can be expressed in terms of pheno-genotype frequencies or in terms of separate variant frequencies by the identities: $P_{\mathrm{AM}}=p_{\mathrm{A}} p_{\mathrm{M}}+D, P_{\mathrm{Am}}=p_{\mathrm{A}}\left(1-p_{\mathrm{M}}\right)-D$, $P_{\mathrm{aM}}=\left(1-p_{\mathrm{A}}\right) p_{\mathrm{M}}-D$, where $D$ is the linkage disequilibrium after reproduction but before recombination and cultural transmission, and is defined by

$D=P_{\mathrm{AM}} P_{\mathrm{am}}-P_{\mathrm{Am}} P_{\mathrm{aM}}$

(e.g., Bürger (2000) and Ewens (2004), note that $D$ is distinct from $\operatorname{Cov}\left[p_{\mathrm{A}(\mathrm{iN})}, p_{\mathrm{M}(\mathrm{iN})}\right]$, which can be interpreted as the linkage disequilibrium after cultural transmission). Hence, to ascertain the dynamics of the frequencies $p_{\mathrm{A}}$ and $p_{\mathrm{M}}$ in closed form we also need the dynamics of the linkage disequilibrium. We computed the dynamics of the linkage disequilibrium from the dynamics of the pheno-genotypes in the population (Eq. (A.3) in the Appendix). With the linkage disequilibrium, we now have all the elements necessary to determine the evolutionary dynamics of the helping and modifier variants.

\section{Results}

\subsection{Co-evolution of helping and one-to-many transmission}

\subsubsection{Helping variant}

We present explicit expressions for the moments of variant $A$ frequency appearing in the Price equation in the Appendix (see Eqs. (A.8)-(A.11)). When the frequency of variant $M$ is zero, the vertical transmission variant $m$ is fixed in the population, and the linkage disequilibrium $D$ and all covariances will be zero; hence $R_{\mathrm{AM} \cdot \mathrm{A}}=0$, $R_{\mathrm{A} / \mathrm{A}}=0$, and $\mathrm{E}\left[\Delta p_{\mathrm{A}(\mathrm{iN})}\right]=0$. Then, from Eq. (4) we see that the change in frequency of the helping variant is

$\bar{w} \Delta p_{\mathrm{A}}=-C_{\mathrm{A}} p_{\mathrm{A}}\left(1-p_{\mathrm{A}}\right)$. 
Hence, when the transmission of helping is strictly vertical $\left(p_{\mathrm{M}}=\right.$ 0 ) and helping results in a direct fitness cost to the actor (here when $C_{\mathrm{A}}>0$ ), altruistic helping, defined sensu Hamilton $(1964,1970)$ from effects on direct fitness, is counter-selected at all variant frequencies under random group formation. This is a standard result (Hamilton, 1975; Cohen and Eshel, 1976; Charlesworth, 1979; Uyenoyama and Feldman, 1980; Grafen, 1984), and is a direct consequence of the fact that relatedness is zero in that case. By contrast, whenever the transmission of the helping variant $A$ is not strictly vertical $\left(p_{\mathrm{M}}>0\right)$, the cultural relatedness $R_{\mathrm{A} / \mathrm{A}}$ between group members becomes positive, and indirect benefits affect the evolutionary dynamics. Although relatedness decreases with increasing group size, it remains positive even when group size becomes very large $(N \rightarrow \infty)$, when it takes the value (see Appendix Eqs. (A.9) and (A.11))

$R_{\mathrm{A} / \mathrm{A}}=\frac{p_{\mathrm{A}}\left(1-p_{\mathrm{A}}\right) p_{\mathrm{M}}^{2}}{\left(p_{\mathrm{A}}-(1-r) D\right)\left(1-p_{\mathrm{A}}+(1-r) D\right)}$.

Similarly, expression of variant $M$ produces an association between variant $A$ and $M$ in the same individual, with the result that under large group size ( $N \rightarrow \infty$, see Appendix Eqs. (A.9) and (A.10))

$R_{\mathrm{AM} \cdot \mathrm{A}}=\frac{(1-r) D p_{\mathrm{M}}}{\left(p_{\mathrm{A}}-(1-r) D\right)\left(1-p_{\mathrm{A}}+(1-r) D\right)}$.

Although variant $M$ causes individuals bearing variant $A$ to interact with each other (i.e., it generates a positive $R_{\mathrm{A} / \mathrm{A}}$ ), it also decreases the frequency of $A$ in the population. This occurs whenever variants $A$ and $M$ are associated within individuals because those bearing $M$ may adopt the defector variant $a$. From Eq. (A.8) in the appendix, the expected change in variant frequency solely due to horizontal cultural transmission is given by

$\mathrm{E}\left[\Delta p_{\mathrm{A}(\mathrm{iN})}\right]=-\frac{(1-r) D(N-1)}{N}$,

and is negative provided variant $A$ is associated with variant $M$ $(D>0)$, which is likely to be the case provided the cost $C_{M}$ of the modifier is not too high (see Eqs. (A.15) and (A.17)).

Inserting Eqs. (15)-(17) into Eq. (4) we find that the change in frequency of $A$ when groups are of large size $(N \rightarrow \infty)$ is given by

$$
\begin{aligned}
\bar{w} \Delta p_{\mathrm{A}}= & p_{\mathrm{A}}\left(1-p_{\mathrm{A}}\right)\left(-C_{\mathrm{A}}+B p_{\mathrm{M}}^{2}\right) \\
& -(1-r) D\left(1-D B(1-r)-C_{\mathrm{A}}\left(1-p_{\mathrm{A}}\right)+B p_{\mathrm{A}}\right) .
\end{aligned}
$$

Thus, selection may favor the helping variant $A$ if the frequency $p_{M}$ of $M$ in the population is high enough that the right-hand side of Eq. (18) is positive.

\subsubsection{Modifier variant}

When the frequency of variant $A$ is zero, the linkage disequilibrium $D$ will be zero and all covariances will be zero in which case $R_{\mathrm{AM} \cdot \mathrm{M}}=0$ and $R_{\mathrm{A} / \mathrm{M}}=0$. Then, Eq. (7) informs us that the change in frequency of the modifier variant is simply given by $\bar{w} \Delta p_{M}=-C_{M} p_{M}\left(1-p_{M}\right)$. Thus, when variant $M$ is costly, it is counter-selected at all variant frequencies. When the helping variant $A$ is introduced into the population, variants $A$ and $M$ become positively associated in different individuals within groups. This association is given by $R_{\mathrm{A} / \mathrm{M}}=(1-r) D /\left[N\left(1-p_{\mathrm{M}}\right)\right]$ (see Appendix Eqs. (A.12) and (A.13)) and causes indirect selection on the modifier variant $M$, which may then increase in frequency in the population. Inserting Eqs. (A.10), (A.12) and (A.13) into Eq. (7), we find that the change in frequency of $M$ is given by

$$
\begin{aligned}
\bar{w} \Delta p_{\mathrm{M}}= & -C_{\mathrm{M}} p_{\mathrm{M}}\left(1-p_{\mathrm{M}}\right)+(1-r) \\
& \times D\left(\frac{B p_{\mathrm{M}}}{N}-C_{\mathrm{A}}\left(p_{\mathrm{M}}+\frac{1-p_{\mathrm{M}}}{N}\right)\right) .
\end{aligned}
$$

Thus, selection may favor the modifier variant $M$ if the benefit $B$ of helping is large enough that the right-hand side of Eq. (18) is positive. However, the indirect selective pressure on the variant $M$ is rather weak because it depends on the inverse of group size multiplied by the linkage disequilibrium $D$, which is usually small. Consequently, the benefits of helping will only weakly affect the evolutionary dynamics of the modifier variant $M$. In groups of very large size $(N \rightarrow \infty)$, Eq. (19) becomes

$\bar{w} \Delta p_{\mathrm{M}}=-C_{\mathrm{M}} p_{\mathrm{M}}\left(1-p_{\mathrm{M}}\right)-C_{\mathrm{A}} p_{\mathrm{M}}(1-r) D$.

The modifier variant $M$ will therefore never spread in groups of very large size when it produces a cost $\left(C_{M}>0\right)$. This is in contrast to the helping variant $A$ which may still be selected for in this case (see Eq. (18)). Finally, in order to obtain the evolutionary dynamics of the helping and modifier variants explicitly, we need the dynamics of the linkage disequilibrium $D$, which unfortunately is very complicated, and we present only special cases in the Appendix (see Eqs. (A.15) and (A.17)).

\subsubsection{Local stability}

Co-evolution of the frequency $p_{\mathrm{A}}$ of variant $A$, the frequency $p_{\mathrm{M}}$ of variant $M$ and the linkage disequilibrium $D$ occurs via a dynamical system with three state variables. In order to ascertain whether helping and horizontal transmission may invade the population when rare, we carried out a local stability analysis of the equilibrium point $\left(p_{\mathrm{A}}=0, p_{\mathrm{M}}=0, D=0\right)$. Standard methods show that the three eigenvalues of the linearized dynamical system at this point are given by: $1-C_{\mathrm{M}}, 1-C_{\mathrm{A}}$ and $(1-r)\left(1-C_{\mathrm{A}}-C_{\mathrm{M}}\right) / N$. Hence, if both the helping and the modifier variant produce a cost, all eigenvalues are smaller than unity. When all eigenvalues of a linearized dynamical system are smaller than unity at a given point in state space, this point is locally stable, which implies here that rare costly variants $A$ and $M$ cannot invade the population. In order to establish whether a population of helpers with horizontal transmission is immune to defection, we carried out a local stability analysis of the equilibrium point $\left(p_{\mathrm{M}}=1\right.$, $\left.p_{\mathrm{A}}=1, D=0\right)$. The three eigenvalues of the linearized dynamical system at this point are given by: $\left(1+B-C_{A}\right) /\left(1+B-C_{A}-C_{M}\right)$, $\left(1-C_{\mathrm{M}}\right) /\left(1+B-C_{\mathrm{A}}-C_{\mathrm{M}}\right)$ and $(1-r)(1+B(1-1 / N)) /\left(1+B-C_{\mathrm{A}}-C_{\mathrm{M}}\right)$. Hence, if both the helping and the modifier variant produce a cost, at least one eigenvalue is greater than unity, and the equilibrium point $\left(p_{\mathrm{A}}=1, p_{\mathrm{M}}=1, D=0\right)$ is locally unstable. Thus, the vertical transmission variant $m$ can invade the population, which, in turn, may allow for the invasion of the defector variant $a$. This local stability analysis suggests that for the frequency of the helping variant $A$ to increase in frequency when rare, and achieve fixation, the modifier variant $M$ must either be neutral $\left(C_{M}=0\right)$ or result in a direct benefit $\left(C_{M}<0\right)$ due to extrinsic factors.

\subsubsection{Dynamics}

In Fig. 2 we illustrate the dynamics of the frequencies of variants $A$ and $M$, and the components of the Price equations $\left(R_{\mathrm{AM} \cdot \mathrm{A}}, R_{\mathrm{A} / \mathrm{A}}\right.$, $R_{\mathrm{AM} \cdot \mathrm{M}}, R_{\mathrm{A} / \mathrm{M}}$ and $\left.\mathrm{E}\left[\Delta p_{\mathrm{A}(i \mathrm{~N})}\right]\right)$ as functions of time under different sets of parameter values. When variant $A$ and $M$ are initially not rare in the population (here we used $p_{A}=p_{M}=0.1$ ), and the modifier variant $M$ results in a fitness cost to the actor $\left(C_{\mathrm{M}}>0\right)$, selection decreases the frequency of the modifier variant (Fig. 2A). This induces a steady decline of the associations between variant $A$ in different individuals from the same group, which ultimately drives the helping variant $A$ to extinction, although the dynamics go through a transient phase in which the frequency of $A$ first increases and then declines when the cost of bearing the modifier is low (Fig. 2A). For the same set of parameter values but with a neutral modifier variant $\left(C_{M}=0\right)$, the helping variant $A$ is driven to fixation if the cost-to-benefit ratio of helping is small (Fig. 2B), but is counter-selected if the cost-to-benefit 
A

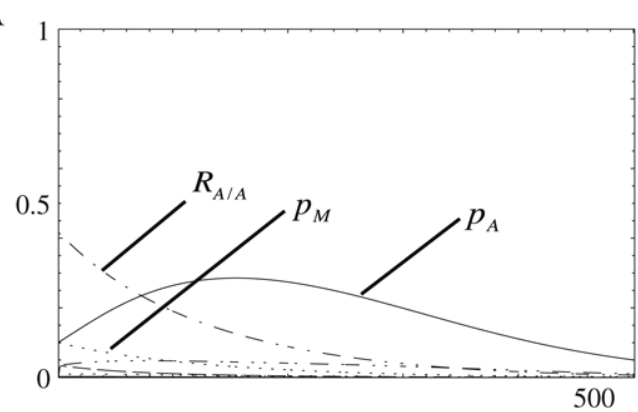

B

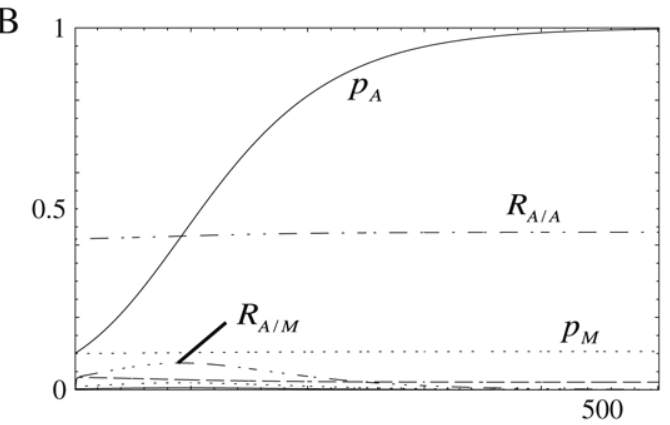

C

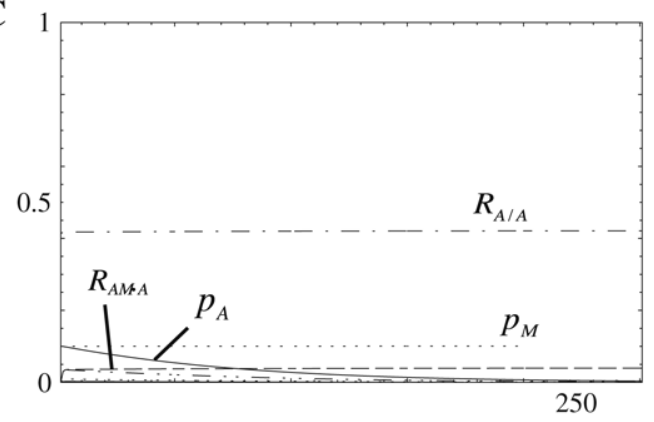

$\mathrm{D}$

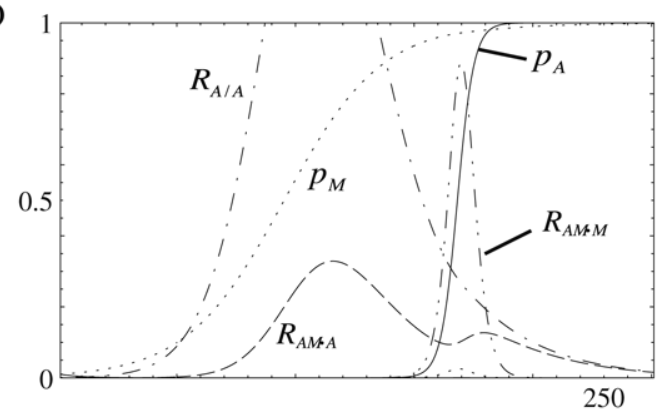

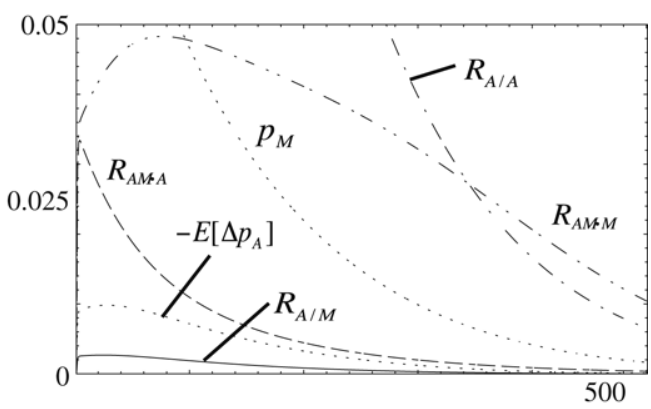
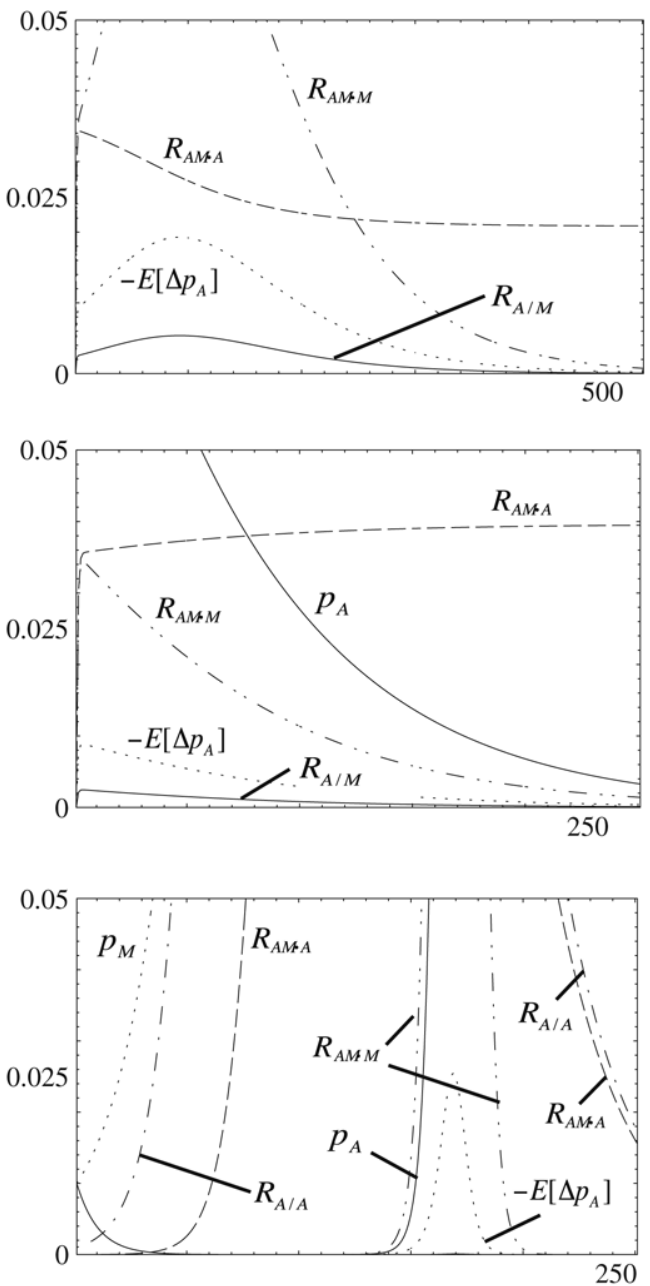

Generations

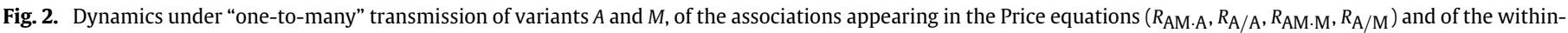

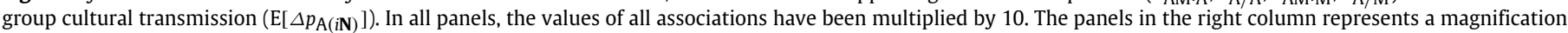

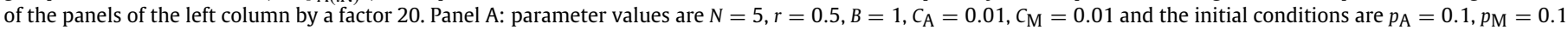

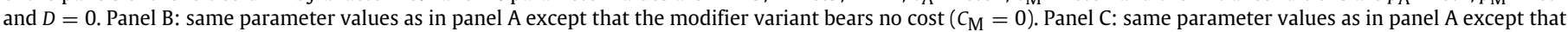

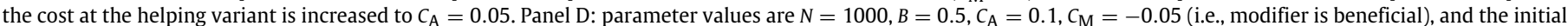
conditions are $p_{\mathrm{A}}=0.01, p_{\mathrm{M}}=0.01$ and $D=0$.

ratio becomes large (Fig. 2C). In all of our numerical simulations with a neutral modifier $M$, the frequency of $M$ remained nearly constant while variant $A$ either invaded or was expelled from the population. Thus, the hitchhiking effects are very small and the associations between variants $A$ and $M$ in the same and in different individuals will remain approximately constant during the invasion or expulsion of $A$. Finally, when variant $M$ results in a direct fitness benefit $\left(C_{M}<0\right)$, both helping and non-vertical transmission can co-evolve from initially very low frequencies (i.e., rare mutant can invade). The modifier variant $M$ invades the population first (Fig. 2D), markedly increasing the relatedness $R_{\mathrm{A} / \mathrm{A}}$ during its sweep through the population, which then drives variant $A$ to fixation.

\subsection{Co-evolution of helping and conformist transmission}

Unfortunately, we found no analytical solutions for the conformist transmission model Eq. (A.2), and we were unable to carry out the local stability analysis analytically. We therefore numerically iterated the recursions for pheno-genotype frequencies to 
A

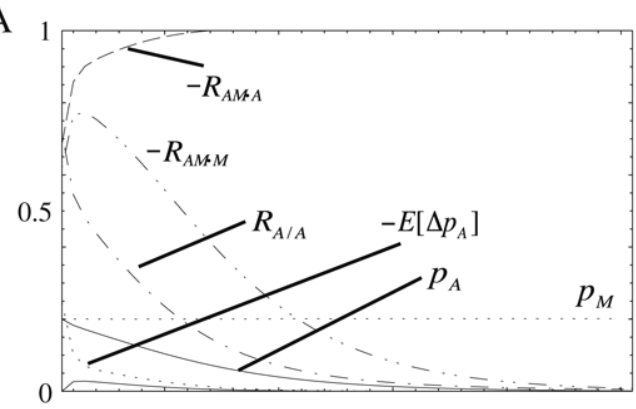

B

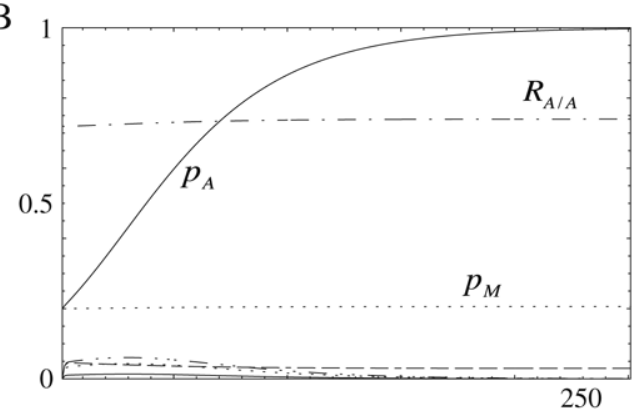

$\mathrm{C}$

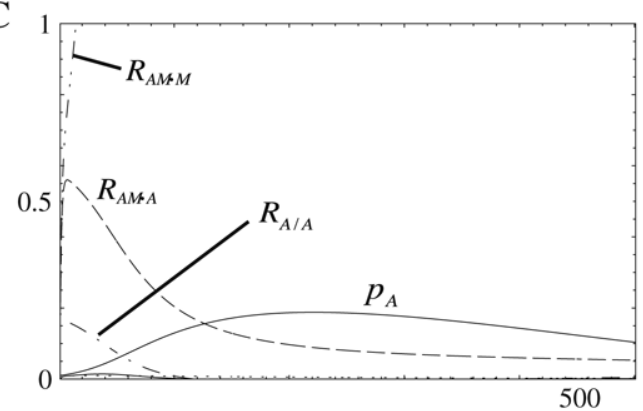

D

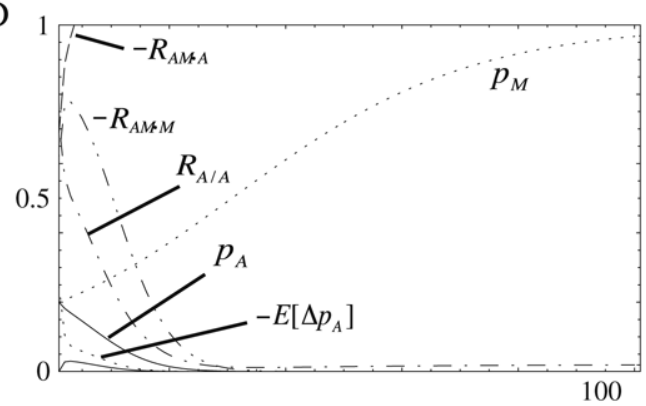

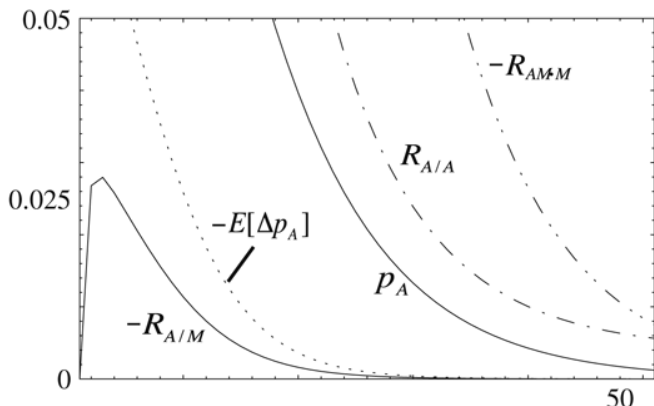
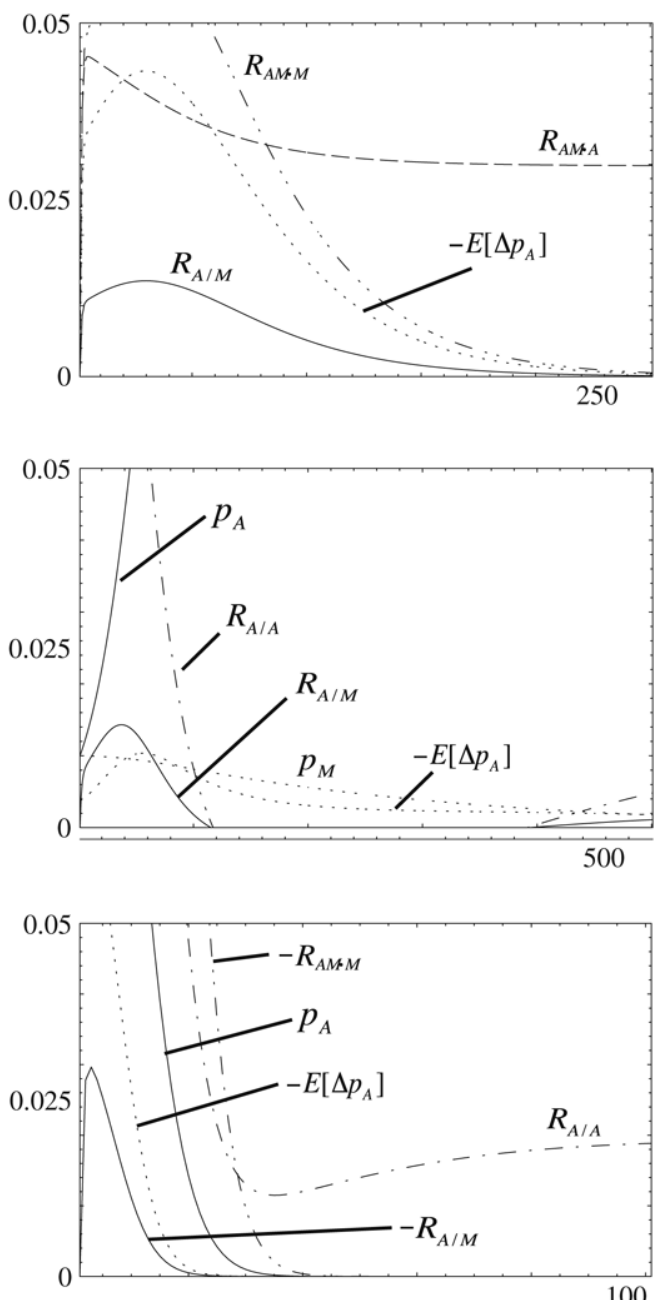

Generations

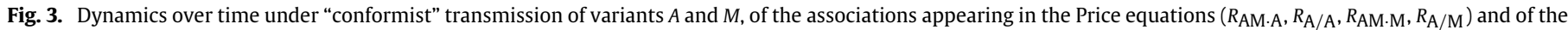

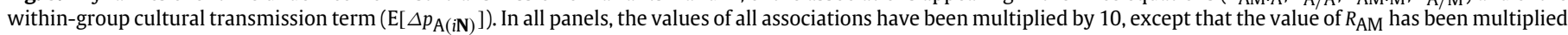

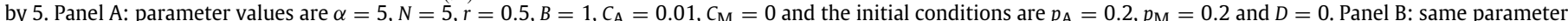

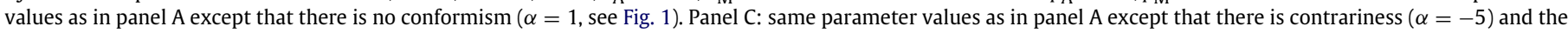
initial conditions are $p_{\mathrm{A}}=0.01, p_{\mathrm{M}}=0.01$ and $D=0$. Panel $\mathrm{D}$ : same parameter values as in panel A except that the modifier results in a direct benefit $C_{\mathrm{M}}=-0.05$.

study the dynamics of this model Eq. (A.3). In Fig. 3 we illustrate the dynamics of the frequencies of the helping and modifier variants, and the components of the Price equations $\left(R_{\mathrm{AM} \cdot \mathrm{A}}, R_{\mathrm{A} / \mathrm{A}}, R_{\mathrm{AM} \cdot \mathrm{M}}\right.$, $R_{\mathrm{A} / \mathrm{M}}$ and $\left.\mathrm{E}\left[\Delta p_{\mathrm{A}(\mathrm{iN})}\right]\right)$ as functions of time under different sets of parameter values for the conformist transmission rule. If the modifier variant $M$ results in a fitness cost or is neutral $\left(C_{M} \geq 0\right)$, it causes the helping variant $A$ to be expelled from the population under biased conformism $(\alpha=5)$, even with high initial frequencies of variants $A$ and $M$ (by high initial frequencies of a variant $K$ we mean here and below $p_{\mathrm{K}} \gg 0$ and $p_{\mathrm{K}}<0.5$, and we used
$p_{A}=p_{M}=0.2$ in Fig. $3 A$ ). The helping variant $A$ is counter-selected because individuals bearing variant $M$ adopt the most frequent variant in their group, namely the defector $a$. This results in strong pressure against $A$ causing the within-group cultural transmission term of the Price equation $\left(\mathrm{E}\left[\Delta p_{\mathrm{A}(\mathrm{iN})}\right]\right)$ to be much more negative under conformist transmission than under one-to-many transmission (compare the right panels of Figs. $2 \mathrm{~B}$ and $3 \mathrm{~A}$ ). It is this strong negative value of the cultural transmission term $\left(\mathrm{E}\left[\Delta p_{\mathrm{A}(\mathrm{iN})}\right]\right)$ that drives variant $A$ out of the population, even if cultural relatedness is initially high. Hence, while the stochastic fluctuations of variant 
frequencies due to finite group size seem to generate high relatedness under conformist transmission, even if the frequency of $A$ is initially rare, it is the negative term $\mathrm{E}\left[\Delta p_{\mathrm{A}(i \mathbf{N})}\right]$ that prevents the evolution of costly variant $A$ when rare.

Moreover, conformist transmission results in negative statistical associations of the helping and modifier variants within and between individuals $\left(R_{\mathrm{AM} \cdot \mathrm{M}}<0\right.$ and $R_{\mathrm{A} / \mathrm{M}}<0$, Fig. $\left.3 \mathrm{~A}\right)$ because individuals bearing variant $M$ adopt primarily the defector variant $a$ when $p_{\mathrm{A}}<0.5$. That conformist transmission has an adverse affect on the evolution of helping can best be seen by tuning the parameter $\alpha$ that determines the conformist bias (Fig. 1). Under unbiased conformism $(\alpha=1)$, individuals copy the strategies with a probability equal to their frequencies in groups, and the helping variant $A$ actually evolves for a set of parameter values under which it is counter-selected with biased conformist transmission (Fig. 3B). Note that when $\alpha=1$ the transmission mode has some similarity with one-to-many transmission because in both cases individuals adopt strategies according to their frequency in groups in an unbiased way (i.e., the leader will carry variant $A$ with a probability equal to its frequency in the group). The difference is that under one-to-many transmission all individuals with variant $M$ adopt the helping variant from the same individual, while with unbiased conformist transmission, each individual carrying $M$ chooses independently, which results in a lower relatedness between group members. A large number of repeated rounds of horizontal transmission with $\alpha=1$ would, in fact, be equivalent to the one-to-many transmission rule, and result in the fixation of a helping variant at the level of the group in a single round of horizontal transmission. If there is contrariness $(\alpha=-5)$, variant $A$ increases very rapidly at first but is counter-selected later because the modifier variant $M$ is outrun by the vertical transmission variant $m$ (Fig. 3C). This occurs because under contrariness, relatedness decreases very rapidly while the linkage disequilibrium builds up, resulting in indirect selection pressure on variant $M$, which is then counter-selected. Finally, when conformist transmission results in a direct benefit through some extrinsic factor $\left(C_{M}<0\right)$, variant $M$ increases and causes the frequency of $A$ to decline very rapidly (Fig. 3D).

\section{Discussion}

Cultural inheritance of helping behaviors has been proposed to be more favorable to the evolution of altruistic helping in groups of large size than genetic inheritance (Boyd and Richerson, 1982; Fehr et al., 2002; Henrich, 2004; Richerson and Boyd, 2005; Boyd and Richerson, 2005). Horizontal and oblique cultural transmission can markedly increase similarities within groups and differences between groups relative to those stemming from genetic transmission. This occurs because under cultural transmission many individuals can easily adopt the variants from only a few individuals, which can produce high associations between the cultural variants carried by individuals within groups (i.e., high cultural inbreeding sensu (Cavalli-Sforza and Feldman, 1981 , p. 352) or cultural relatedness sensu (Allison, 1991)), thereby increasing the selective pressure on helping behaviors. In this paper we studied the co-evolution of culturally-inherited helping traits and two assimilation rules under horizontal cultural transmission: one that is frequency-independent but class-based (one-to-many transmission) and one that is frequency-dependent (conformist transmission). We assumed a panmictic population in order to rule out any effect of limited migration, which inevitably leads to identity by descent within groups, and would by itself increase the cultural and/or the genetic relatedness between group members. In the absence of cultural transmission, altruistic helping can never evolve in our population setting Eq. (14), and we asked whether natural selection can drive the evolution of an inheritance system that is more susceptible to co-evolution with altruistic helping.

Our models show that altruistic helping can evolve in groups of very large size when helping is inherited culturally rather than genetically. For instance, under a one-to-many assimilation rule, the cultural relatedness remains positive in groups of large size even under complete migration. This illustrates that cultural inheritance can produce positive relatedness coefficients between group members, which are independent of group size and migration rate (Eq. (15), see also Lehmann et al. (2007, in press)). Under cultural inheritance, it is no longer the ecological or demographic processes of the population that primarily determine the relatedness between interacting individuals at the helping locus, but the specificities of the individual assimilation rules, which can involve vertical, horizontal and oblique cultural transmissions. Our one-to-many transmission model is only one example of a more general situation where the assimilation rule of cultural variants is frequency-independent but classor status-based (and there is no association between variants and class), which can result in high cultural relatedness at all variant frequencies because some individuals contribute more than others to the transmission process (see also Cavalli-Sforza and Feldman (1981, pp. 315-339)). For instance, there is empirical evidence that individuals may copy others with higher wealth or prestige (Rogers, 1995), which are particular examples of class structured population. Here, we can make a direct analogy with systems of genetic inheritance, where class structured populations generally result in a higher variance in reproductive success, leading to increased local genetic drift (Rousset, 2004), which favors the evolution of altruistic helping.

While a rare helping variant can invade a population of defectors under one-to-many transmission and become fixed when it is common (Fig. 2B), the same helping variant is counter-selected under biased conformist transmission, when everything else is held constant (compare Figs. 2B with 3A). Because conformist transmission causes individuals to copy the most frequent variant in a group, a rare helping variant is strongly disfavored under this transmission rule. As can be seen from Fig. 3A, even at high initial frequencies (here $p_{\mathrm{A}}=0.2$; by high initial frequencies we mean $p_{\mathrm{A}} \gg 0$ and $\left.p_{\mathrm{A}}<0.5\right)$ and with low cost-to-benefit ratio, the helping variant $A$ is ousted from the population. Fig. $3 \mathrm{~A}$ shows that while biased conformist transmission may result in high relatedness $R_{\mathrm{A} / \mathrm{A}}$ between group members, it creates a strong negative change in the frequency of the helping variant due to within-group cultural transmission $\left(\mathrm{E}\left[\Delta p_{\mathrm{A}(\mathrm{iN})}\right]<0\right.$ in Eq. (4)). This by-product of conformist transmission causes negative biased transmission and forces the costly helping variant to be expelled from the population. We emphasize here that the stochastic fluctuations of variant frequencies caused by finite group size entail that a helping variant can be locally common while globally rare (which can generate high $R_{\mathrm{A} / \mathrm{A}}$ even if the frequency of $A$ is globally low). Consequently, our model offers in principle a stronger case for the evolution of helping through biased conformist transmission than the previous ones (Boyd and Richerson, 1982; Henrich and Boyd, 2001), which assumed limited migration but infinite group size, a situation that cannot produce a positive cultural relatedness at low variant frequencies through random sampling of variant frequencies. But even when stochastic fluctuations in variant frequencies do occur, it follows from our result that the withingroup transmission term of the Price equation ( $\mathrm{E}\left[\Delta p_{\mathrm{A}(i \mathrm{~N})}\right]$ in Eq. (4)) can have a very strong adverse effect on the evolution of culturallyinherited helping behaviors. On the other hand, when cultural transmission occurs by unbiased conformist transmission $(\alpha=1$ in Fig. 1), the negative selective pressure on a rare helping variant is relaxed and relatedness among group members increases. This 
situation allows a rare helping variant $A$ to sweep to fixation in the population (Fig. 3B). While our results show that biased conformist transmission does not facilitate the spread of costly behaviors unless they have already reached a very high frequency in the population $\left(p_{\mathrm{A}}>0.5\right)$ due to some extrinsic factors, they do not imply that all rare variants would be eliminated by conformist transmission. Indeed, if variant $A$ resulted in some direct benefit $\left(C_{\mathrm{A}}<0\right)$, Eq. (4) shows that $A$ could invade when rare because one can always find a value of $C_{A}$ that overcomes the adverse effect of biased cultural transmission as measured by $\mathrm{E}\left[\Delta \mathrm{p}_{\mathrm{A}(\mathrm{iN})}\right]$.

Our results illustrate that a frequency-dependent assimilation rule such as biased conformist transmission, which is often invoked in discussions on the evolution of helping in humans (Boyd and Richerson, 1982; Henrich and Boyd, 2001; Richerson and Boyd, 2005; Henrich, 2004), is unlikely to promote the evolution of altruistic helping in situations where it is otherwise difficult to explain, that is, in populations of large size when the trait is initially rare. On the other hand, a frequency-dependent assimilation rule such as "contrariness transmission" favors the helping variant when rare but opposes its fixation. This all suggests that frequency-dependent assimilation rules are unlikely to help to explain both the emergence and stability of culturallyinherited helping traits, unless one postulates a combination of the conformist and contrariness effects. By contrast, frequencyindependent assimilation rules based on classes or stratification can result in high cultural relatedness at all variant frequencies, while simultaneously having only minimal impact on the withingroup transmission rate (here $\mathrm{E}\left[\Delta p_{\mathrm{A}(\mathrm{iN})}\right]$ ). These assimilation rules allow for both the emergence of costly helping when rare and its stability when common.

In our models, the helping variant $A$ cannot evolve without the modifier variant $M$, but the modifier variant hitchhikes only weakly with the helping variant. Further, helping and the assimilation rules also cannot co-evolve when both variants are initially rare, unless there is direct selection on the modifier (see section "Local stability"). This suggests that the benefits of helping are unlikely to be the main forces causing the evolution of nonvertical transmission in panmictic populations (i.e., random group formation). The evolution of a psychological propensity to copy the leader or the majority is thus not explained directly by our models. But oblique or horizontal transmission may evolve through other factors such as temporally varying environments (Wakano et al., 2004; Wakano and Aoki, 2006). Once social learning is introduced in the population through extrinsic factors, that result in direct selection on the modifier variant $M$, the flow of information within groups becomes more skewed than under genetic transmission, which may then drive the increase of a rare costly helping variant in groups of any size (see section "Local stability"). Although we assumed a panmictic population, different demographic regimes might result in different co-evolutionary dynamics between helping and cultural transmission. For instance, under both limited migration and finite group size, linkage disequilibrium due to identity by descent develops between loci in the same and in different individuals, possibly increasing indirect selection on a modifier variant.

A distinctive feature of cultural transmission relative to genetic transmission of helping behaviors is the ease with which traits can be passed on to biologically unrelated individuals through oblique or horizontal transmission, a process that can lead to high cultural relatedness between individuals within groups. But under both cultural and genetic inheritance, it is the evolutionary dynamics that selects the equilibrium strategy(ies) for the social game(s) played by the individuals in the population, irrespective of individuals having any understanding or knowledge of the game they are playing. In other words, individuals cannot individually learn how to play the social game through trial and error, insight or deduction. Humans, however, are not immutably programmed by their culture with fixed behaviors, but express behavioral flexibility within and across social games (Binmore, 2006; Binmore and Swierzbinski, 2006). Indeed, humans have the ability to learn individually (Wakano et al., 2004; Richerson and Boyd, 2005; Borenstein et al., 2008), which suggests that it would be useful to consider both individual and social learning of helping behaviors jointly in future research.

\section{Acknowledgments}

We thank Kevin Foster for the helpful comments on the manuscript. LL is supported by a grant from the Swiss NSF. This research is supported by NIH grant GM28016.

\section{Appendix}

\section{A.1. Cultural transmission kernels}

We derive here the distribution of group type after cultural transmission given that the distribution of group type before cultural transmission is given by Eq. (11). Under the one-tomany transmission situation, all individuals bearing variant $M$ in a group copy the cultural variant at the helping locus of a randomly sampled individual from their group (the leader or the teacher of the group). If a group consists of pheno-genotype array $\left(N_{\mathrm{AM}}^{\star}=k, N_{\mathrm{Am}}^{\star}=i, N_{\mathrm{aM}}^{\star}=h-k\right)$ before cultural transmission, the probability that the randomly chosen leader bears variant $A$ is $(k+i) / N$. When this event occurs, the group becomes of type $\left(N_{\mathrm{AM}}=h, N_{\mathrm{Am}}=i, N_{\mathrm{aM}}=0\right)$ after cultural transmission. With complementary probability $1-(k+i) / N$ the randomly chosen leader bears variant $a$, in which case the group becomes of type $\left(N_{\mathrm{AM}}=0, N_{\mathrm{Am}}=i, N_{\mathrm{aM}}=h\right)$ after cultural transmission. With this, the group type distribution after cultural transmission is

$$
\begin{aligned}
& \operatorname{Pr}\left(N_{\mathrm{AM}}=h, N_{\mathrm{Am}}=i, N_{\mathrm{aM}}=j\right) \\
& =\left\{\begin{array}{l}
\sum_{k=0}^{h} \frac{k+i}{N} \operatorname{Pr}\left(N_{\mathrm{AM}}^{\star}=k, N_{\mathrm{Am}}^{\star}=i, N_{\mathrm{aM}}^{\star}=h-k\right) \\
\text { for } h>0 \text { and } j=0 \\
\sum_{k=0}^{j} \frac{N-(k+i)}{N} \operatorname{Pr}\left(N_{\mathrm{AM}}^{\star}=k, N_{\mathrm{Am}}^{\star}=i, N_{\mathrm{aM}}^{\star}=j-k\right) \\
\text { for } h=0 \text { and } j>0 \\
\operatorname{Pr}\left(N_{\mathrm{AM}}^{\star}=0, N_{\mathrm{Am}}^{\star}=i, N_{\mathrm{aM}}^{\star}=0\right) \\
\text { for } h=0 \text { and } j=0,
\end{array}\right.
\end{aligned}
$$

which satisfies $\sum_{h=0}^{N} \sum_{i=0}^{N-h} \sum_{j=0}^{N-h-i} \operatorname{Pr}\left(N_{\mathrm{AM}}=h, N_{\mathrm{Am}}=i, N_{\mathrm{aM}}=j\right)=$ 1.

Under conformist transmission, each individual bearing variant $M$ independently copies the cultural variant of an individual in its group. Hence, the probability that $h$ individuals bearing variant $M$, among a total number of $h+j$ such individuals, have adopted variant $A$ will be obtained from the Binomial distribution. The distribution of group types can then be written as

$$
\begin{aligned}
& \operatorname{Pr}\left(N_{\mathrm{AM}}=h, N_{\mathrm{Am}}=i, N_{\mathrm{aM}}=j\right) \\
& =\sum_{k=0}^{h+j} \frac{(h+j) !}{h ! j !} u\left(x_{\mathrm{A}}^{\star}\right)^{h}\left(1-u\left(x_{\mathrm{A}}^{\star}\right)\right)^{j} \\
& \quad \times \operatorname{Pr}\left(N_{\mathrm{AM}}^{\star}=k, N_{\mathrm{Am}}^{\star}=i, N_{\mathrm{AM}}^{\star}=h+j-k\right),
\end{aligned}
$$

where $u\left(x_{\mathrm{A}}^{\star}\right)$ is the conformist transmission function Eq. (1), which depends on the frequency $x_{\mathrm{A}}^{\star}=(k+i) / N$ of variant $A$ in a group before cultural transmission.

\section{A.2. Genotype dynamics}

In order to evaluate the components of the Price equations explicitly, we need expressions over time for the frequencies of 
the pheno-genotypes in the population. The recursion for the frequency of pheno-genotype $k$ can be written as

$P_{k}^{\prime}=\frac{1}{\bar{w}} \sum_{\mathbf{N}} f_{k}(\mathbf{N}) w_{k}(\mathbf{N}) \operatorname{Pr}(\mathbf{N})$,

where the prime denotes the next generation, $f_{k}(\mathbf{N})$ is the frequency of pheno-genotype $k$ in a group of type $\mathbf{N}$ and $w_{k}(\mathbf{N})$ is its corresponding fitness. The frequencies of the pheno-genotypes, conditional on group type, are given by

$$
\begin{aligned}
& f_{\mathrm{AM}}\left(N_{\mathrm{AM}}=h, N_{\mathrm{Am}}=i, N_{\mathrm{aM}}=j\right)=\frac{h}{N} \\
& f_{\mathrm{Am}}\left(N_{\mathrm{AM}}=h, N_{\mathrm{Am}}=i, N_{\mathrm{aM}}=j\right)=\frac{i}{N} \\
& f_{\mathrm{aM}}\left(N_{\mathrm{AM}}=h, N_{\mathrm{Am}}=i, N_{\mathrm{aM}}=j\right)=\frac{j}{N} \\
& f_{\mathrm{am}}\left(N_{\mathrm{AM}}=h, N_{\mathrm{Am}}=i, N_{\mathrm{aM}}=j\right)=\frac{N-h-i-j}{N},
\end{aligned}
$$

and the fitness functions, corresponding to Eq. (3), are given by

$$
\begin{aligned}
& w_{\mathrm{AM}}\left(N_{\mathrm{AM}}=h, N_{\mathrm{Am}}=i, N_{\mathrm{aM}}=j\right)=1-C_{\mathrm{A}}-C_{\mathrm{M}}+B\left(\frac{h+i-1}{N-1}\right) \\
& w_{\mathrm{Am}}\left(N_{\mathrm{AM}}=h, N_{\mathrm{Am}}=i, N_{\mathrm{aM}}=j\right)=1-C_{\mathrm{A}}+B\left(\frac{h+i-1}{N-1}\right) \\
& w_{\mathrm{aM}}\left(N_{\mathrm{AM}}=h, N_{\mathrm{Am}}=i, N_{\mathrm{aM}}=j\right)=1-C_{\mathrm{M}}+B\left(\frac{h+i}{N-1}\right) \\
& w_{\mathrm{am}}\left(N_{\mathrm{AM}}=h, N_{\mathrm{Am}}=i, N_{\mathrm{aM}}=j\right)=1+B\left(\frac{h+i}{N-1}\right) .
\end{aligned}
$$

\section{A.3. Expectations, variances and covariances}

The expectation, variances and covariances appearing in Eqs. (4) and (7) can be expressed in terms of moments of gene frequencies as $\mathrm{E}\left[\Delta p_{\mathrm{A}(\mathrm{iN})}\right]=\mathrm{E}\left[p_{\mathrm{A}(\mathrm{iN})}\right]-p_{\mathrm{A}}, \operatorname{Var}\left[p_{\mathrm{A}(\mathrm{iN})}\right]=\mathrm{E}\left[p_{\mathrm{A}(\mathrm{iN})}\right](1-$ $\left.\mathrm{E}\left[p_{\mathrm{A}(\mathrm{iN})}\right]\right)$ and $\operatorname{Cov}\left[p_{\mathrm{A}(i \mathbf{N})}, p_{\mathrm{M}(\mathbf{i N})}\right]=\mathrm{E}\left[p_{\mathrm{A}(\mathrm{iN})} p_{\mathrm{M}(i \mathbf{N})}\right]-\mathrm{E}\left[p_{\mathrm{A}(\mathrm{iN})}\right] \mathrm{E}\left[p_{\mathrm{M}(i \mathbf{N})}\right]$. Because $\mathrm{E}\left[p_{\mathrm{A}(-i \mathbf{N})}\right]=\mathrm{E}\left[p_{\mathrm{A}(\mathbf{i N})}\right]$, we also have $\operatorname{Cov}\left[p_{\mathrm{A}(i \mathbf{N})}, p_{\mathrm{A}(-i \mathbf{N})}\right]=$ $\mathrm{E}\left[p_{\mathrm{A}(i \mathbf{N})} p_{\mathrm{A}(-i \mathbf{N})}\right]-\mathrm{E}\left[p_{\mathrm{A}(i \mathbf{N})}\right]^{2}$ and $\operatorname{Cov}\left[p_{\mathrm{M}(i \mathbf{N})}, p_{\mathrm{A}(-i \mathbf{N})}\right]=\mathrm{E}\left[p_{\mathrm{M}(i \mathbf{N})} p_{\mathrm{A}(-i \mathbf{N})}\right]-$ $\mathrm{E}\left[p_{\mathrm{M}(\mathrm{iN})}\right] \mathrm{E}\left[p_{\mathrm{A}(\mathrm{iN})}\right]$. The expectations of products of gene frequencies appearing in these formulae are taken over the group type distribution Eq. (10), and can be expressed as

$$
\begin{aligned}
& \mathrm{E}\left[p_{\mathrm{A}(i \mathbf{N})}\right]=\sum_{h=0}^{N} \sum_{i=0}^{N-h} \sum_{j=0}^{N-h-i} \frac{(h+i)}{N} \operatorname{Pr}\left(N_{\mathrm{AM}}=h, N_{\mathrm{Am}}=i, N_{\mathrm{aM}}=j\right) \\
& \mathrm{E}\left[p_{\mathrm{M}(i \mathbf{N})}\right]=\sum_{h=0}^{N} \sum_{i=0}^{N-h} \sum_{j=0}^{N-h-i} \frac{(h+j)}{N} \operatorname{Pr}\left(N_{\mathrm{AM}}=h, N_{\mathrm{Am}}=i, N_{\mathrm{aM}}=j\right) \\
& \mathrm{E}\left[p_{\mathrm{A}(\mathrm{iN})} p_{\mathrm{M}(\mathrm{iN})}\right]=\sum_{h=0}^{N} \sum_{i=0}^{N-h} \sum_{j=0}^{N-h-i} \frac{h}{N} \operatorname{Pr}\left(N_{\mathrm{AM}}=h, N_{\mathrm{Am}}=i, N_{\mathrm{aM}}=j\right) \\
& \mathrm{E}\left[p_{\mathrm{A}(\mathrm{iN})} p_{\mathrm{A}(-i \mathbf{N})}\right]=\sum_{h=0}^{N} \sum_{i=0}^{N-h} \sum_{j=0}^{N-h-i} \frac{(h+i)(h+i-1)}{N(N-1)} \\
& \times \operatorname{Pr}\left(N_{\mathrm{AM}}=h, N_{\mathrm{Am}}=i, N_{\mathrm{aM}}=j\right) \\
& \mathrm{E}\left[p_{\mathrm{M}(i \mathbf{N})} p_{\mathrm{A}(-i \mathbf{N})}\right]=\sum_{h=0}^{N} \sum_{i=0}^{N-h} \sum_{j=0}^{N-h-i} \frac{h(h-1+i)+j(h+i)}{N(N-1)} \\
& \times \operatorname{Pr}\left(N_{\mathrm{AM}}=h, N_{\mathrm{Am}}=i, N_{\mathrm{aM}}=j\right),
\end{aligned}
$$

where the first term in the ratio of the last equation accounts for the case where a randomly sampled individual carries phenogenotype $A M$ (probability $h / N$ ), in which case another randomly sampled individual (without replacement) carries variant $A$ with probability $(h-1+i) /(N-1)$, while the second term accounts for the case where a randomly sampled individual carries phenogenotype $a M$ (probability $j / N$ ), in which case another randomly sampled individual (without replacement) carries variant $A$ with probability $(h+i) /(N-1)$.

The expectations Eq. (A.6) were calculated either analytically or numerically by using Mathematica (Wolfram, 2003). Using the multinomial distribution as the group type distribution after cultural transmission (so that $\operatorname{Pr}(\mathbf{N})=\operatorname{Pr}\left(\mathbf{N}^{\star}\right)$, see Eqs. (10) and (11)), we recover: $\mathrm{E}\left[p_{\mathrm{A}(\mathrm{iN})}\right]=p_{\mathrm{A}}, \mathrm{E}\left[p_{\mathrm{M}(\mathrm{iN})}\right]=p_{\mathrm{M}}$, $\mathrm{E}\left[p_{\mathrm{A}(i \mathbf{N})} p_{\mathrm{M}(i \mathbf{N})}\right]=X_{\mathrm{AM}}, \mathrm{E}\left[p_{\mathrm{A}(i \mathbf{N})} p_{\mathrm{A}(-i \mathbf{N})}\right]=p_{\mathrm{A}}^{2}, \mathrm{E}\left[p_{\mathrm{M}(i \mathbf{N})} p_{\mathrm{A}(-i \mathbf{N})}\right]=$ $p_{\mathrm{A}} p_{\mathrm{M}}$. Hence, all associations between genes in two different individuals will be zero in this case (i.e., $\operatorname{Cov}\left[p_{\mathrm{A}(\mathrm{iN})}, p_{\mathrm{A}(-i \mathbf{N})}\right]=0$ and $\operatorname{Cov}\left[p_{\mathrm{M}(i \mathbf{N})}, p_{\mathrm{A}(-i \mathbf{N})}\right]=0$ implying that $R_{\mathrm{A} / \mathrm{A}}=0$ and $R_{\mathrm{A} / \mathrm{M}}=$ 0 ). Using Eq. (A.2) as the group type distribution after cultural transmission, we calculated numerically the expectations for the conformist transmission model. Using Eq. (A.1) as the group type distribution after cultural transmission, we evaluated analytically the expectations for one-to-many transmission. We find for the latter case that the frequency of variant $A$ in the population after horizontal cultural transmission is given by

$\mathrm{E}\left[p_{\mathrm{A}(\mathrm{iN})}\right]=p_{\mathrm{A}}-(1-r) D\left(\frac{N-1}{N}\right)$,

where $p_{\mathrm{A}}$ is the frequency of $A$ after reproduction. Thus, the change in frequency of variant $A$ due solely to cultural transmission is

$\mathrm{E}\left[\Delta p_{\mathrm{A}(\mathrm{iN})}\right]=-(1-r) D\left(\frac{N-1}{N}\right)$,

where $(1-r) D$ represents the linkage disequilibrium after recombination and migration but before cultural transmission. The variance in the frequency of $A$ in the population after cultural transmission is then given by

$$
\begin{aligned}
\operatorname{Var}\left[p_{\mathrm{A}(\mathrm{i})}\right]= & \left(p_{\mathrm{A}}-\frac{(1-r) D(N-1)}{N}\right) \\
& \times\left(1-p_{\mathrm{A}}+\frac{(1-r) D(N-1)}{N}\right)
\end{aligned}
$$

and the association between variants $A$ and $M$ in the same individual after cultural transmission is

$\operatorname{Cov}\left[p_{\mathrm{A}(\mathrm{iN})}, p_{\mathrm{M}(\mathrm{i})}\right]=(1-r) D\left(\frac{1}{N}+\left(\frac{N-1}{N}\right) p_{\mathrm{M}}\right)$.

The association between two $A$ variants in different individuals within the same group is given by

$$
\begin{aligned}
\operatorname{Cov}\left[p_{\mathrm{A}(\mathrm{iN})}, p_{\mathrm{A}(-i \mathbf{N})}\right]= & p_{\mathrm{A}}\left(1-p_{\mathrm{A}}\right) p_{\mathrm{M}}\left(\frac{2}{N}+\left(\frac{N-2}{N}\right) p_{\mathrm{M}}\right) \\
& -\left(\frac{(1-r) D}{N}\right)^{2},
\end{aligned}
$$

which depends on the frequencies of the two variants in the population as well as on the linkage disequilibrium. Since the frequency of variant $M$ remains unchanged by horizontal cultural transmission (i.e., $\mathrm{E}\left[p_{\mathrm{M}(\mathrm{iN})}\right]=p_{\mathrm{M}}$ ), the variance in its frequency after cultural transmission is given directly by

$\operatorname{Var}\left[p_{\mathrm{M}(\mathrm{iN})}\right]=p_{\mathrm{M}}\left(1-p_{\mathrm{M}}\right)$,

and the association between variant $A$ and $M$ in two different individuals from the same group is

$\operatorname{Cov}\left[p_{\mathrm{A}(-i \mathbf{N})}, p_{\mathrm{M}(i \mathbf{N})}\right]=\frac{(1-r) D p_{\mathrm{M}}}{N}$.

Finally, from these expressions and Eq. (3), the mean fitness in the population is

$$
\begin{aligned}
\bar{w} & =\mathrm{E}\left[w_{i \mathbf{N}}\right] \\
& =1+\left(B-C_{\mathrm{A}}\right) \mathrm{E}\left[p_{\mathrm{A}(i \mathbf{N})}\right]-C_{\mathrm{M}} \mathrm{E}\left[p_{\mathrm{M}(i \mathbf{N})}\right] \\
& =1+\left(B-C_{\mathrm{A}}\right)\left(p_{\mathrm{A}}-(1-r) D\left(\frac{N-1}{N}\right)\right)-C_{\mathrm{M}} p_{\mathrm{M}} .
\end{aligned}
$$




\section{A.4. Linkage disequilibrium}

The dynamics of the linkage disequilibrium $D$ are complicated. We present here two special cases for one-to-many transmission. First, we give the value of $D$ after one generation when its initial value is zero. This is

$D^{\prime}=\frac{p_{\mathrm{A}}\left(1-p_{\mathrm{A}}\right) p_{\mathrm{M}}\left(1-p_{\mathrm{M}}\right)\left(B G-C_{\mathrm{A}} C_{\mathrm{M}}\right)}{\bar{w}^{2}}$,

where

$G=\frac{1}{N}+\left(\frac{C_{\mathrm{M}}}{N}+\frac{N-2}{N}\right) p_{\mathrm{M}}+\left(B-C_{\mathrm{A}}\right) p_{\mathrm{A}}\left(\frac{1}{N}+\frac{(N-2)}{N} p_{\mathrm{M}}\right)$,

which is strictly positive whenever $N>1$. From these two equations, we see that if the cost $C_{M}$ of the modifier is low, the linkage disequilibrium is likely to become positive over evolutionary time if $B>C_{M}$ and $B>0$. Second, we give the value of $D$ when group size becomes very large $(N \rightarrow \infty)$ and when the cost of the modifier is zero, this is

$$
\begin{aligned}
D^{\prime}= & \frac{p_{\mathrm{M}}}{\bar{w}^{2}}\left[p_{\mathrm{A}}\left(1-p_{\mathrm{A}}\right) p_{\mathrm{M}}\left(1-p_{\mathrm{M}}\right) B\left(1+\left(B-C_{\mathrm{A}}\right) p_{\mathrm{A}}\right)\right. \\
& +(1-r) D\left(1-C_{\mathrm{A}}+B p_{\mathrm{A}}\right)\left(1+B p_{\mathrm{A}}\right) \\
& -(1-r) D B\left(\left(1-p_{\mathrm{A}}\right) p_{\mathrm{A}} p_{\mathrm{M}}\left(B-C_{\mathrm{A}}-B p_{\mathrm{M}}\right)\right. \\
& \left.\left.+D\left(2-C_{\mathrm{A}}+2 B p_{\mathrm{A}}\right)(1-r)-B(1-r)^{2} D^{2}\right)\right],
\end{aligned}
$$

which also suggests that the evolutionary dynamics will result in a positive linkage disequilibrium if $B>C_{M}$ and $B>0$.

\section{References}

Allison, P.D., 1991. Cultural relatedness under oblique and horizontal transmission. Ethology and Sociobiology 13, 153-169.

Aoki, K., 1982. A condition for group selection to prevail over counteracting individual selection. Evolution 36, 832-842.

Axelrod, R., Hammond, R.A., Grafen, A., 2004. Altruism via kin-selection strategies that rely on arbitrary tags with which they coevolve. Evolution 58, 1833-1838.

Binmore, K., 2006. Why do people cooperate? Politics, Philosophy and Economics 5, 81-96.

Binmore, K., Swierzbinski, J., 2006. A little behavioralism can go a long way. In: Does Game Theory Work? The Bargaining Challenge. MIT Press.

Bonner, J.T., 1980. The Evolution of Culture in Animals. Princeton University Press, Princeton, NJ.

Borenstein, E., Aoki, K., Feldman, M.W., 2008. Evolution of learning in fluctuating environments: When selection favors both social and exploratory individuals. Evolution 62, 586-602.

Boyd, R., Richerson, P.J., 1982. Cultural transmission and the evolution of cooperative behavior. Human ecology 10, 325-351.

Boyd, R., Richerson, P.J., 1985. Culture and the Evolutionary Process. University of Chicago Press, Chicago.

Boyd, R., Richerson, P.J., 2005. The Origin and Evolution of Cultures. Oxford University Press, Oxford.

Bshary, R., Bergmuller, R., 2007. Distinguishing four fundamental approaches to the evolution of helping. Journal of Evolutionary Biology 21, 405-420.

Bürger, R., 2000. The mathematical theory of selection, recombination, and mutation. John Wiley and Sons, New York.

Cavalli-Sforza, L., Feldman, M.W., 1973. Models for cultural inheritance. I. Group mean and within group variation. Theoretical Population Biology 4, 42-55.

Cavalli-Sforza, L., Feldman, M.W., 1981. Cultural Transmission and Evolution. Princeton University Press, NJ.

Charlesworth, B., 1979. Note on the evolution of altruism in structured demes. American Naturalist 113, 601-605.

Cohen, D., Eshel, I., 1976. On the founder effect and the evolution of altruistic traits. Theoretical Population Biology 10, 276-302.

Eshel, I., 1972. On the neighbor effect and the evolution of altruistic traits. Theoretical Population Biology 11, 258-277.

Ewens, W.J., 2004. Mathematical Population Genetics. Springer-Verlag, New York.

Fehr, E., Fischbacher, U., Gachter, S., 2002. Strong reciprocity, human cooperation, and the enforcement of social norms. Human Nature 13, 1-25.
Frank, S.A., 1998. Foundations of Social Evolution. Princeton University Press, Princeton, NJ.

Gardner, A., West, S.A., 2006. Demography, altruism, and the benefits of budding. Journal of Evolutionary Biology 19, 1707-1716.

Goodall, J., 1966. Tool using and aimed throwing in a community of free-living chimpanzees. Nature 201, 1264-1266.

Grafen, A., 1984. Natural selection, kin selection and group selection. In: Krebs, J.R. Davies, N. (Eds.), Behavioral Ecology. An Evolutionary Approach. Blackwell Scientific Publications, pp. 62-84.

Grafen, A., 2007a. Detecting kin selection at work using inclusive fitness Proceedings of the Royal Society of London Series B-Biological Sciences 274, 713-719.

Grafen, A., 2007b. An inclusive fitness analysis of a network model of Ohtsuki et al. Journal of Evolutionary Biology 20, 2278-2283.

Hamilton, W.D., 1964. The genetical evolution of social behaviour, I. Journal of Theoretical Biology 7, 1-16.

Hamilton, W.D., 1970. Selfish and spiteful behavior in an evolutionary model. Nature 228, 1218-1220.

Hamilton, W.D., 1971. Selection of selfish and altruistic behaviour in some extreme models. In: Eisenberg, J., Dillon, W. (Eds.), Man and Beast: Comparative Social Behavior. Smithsonian Institutions Press, Washington, DC, pp. 59-91.

Hamilton, W.D., 1975. Innate social aptitudes in man, an approach from evolutionary genetics. In: Fox, R. (Ed.), Biosocial Anthropology. Malaby Press London, pp. 133-157.

Henrich, J., 2004. Cultural group selection, coevolutionary processes and large-scale cooperation. Journal of Economic Behavior and Organization 53, 3-35.

Henrich, J., Boyd, R., 2001. Why people punish defectors - Weak conformist transmission can stabilize costly enforcement of norms in cooperative dilemmas. Journal of Theoretical Biology 208, 79-89.

Jansen, V.A.A., van Baalen, M., 2006. Altruism through beard chromodynamics. Nature 440, 663-666

Le Galliard, J., Ferrière, R., Dieckmann, U., 2003. The adaptive dynamics of altruism in spatially heterogeneous populations. Evolution 57, 1-17.

Lehmann, L., 2006. The evolution of trans-generational altruism: Kin selection meets niche construction. Journal of Evolutionary Biology 20, 181-189.

Lehmann, L., Feldman, M.W., Foster, 2008. Cultural transmission can inhibit the evolution of altruistic helping. American Naturalist (in press).

Lehmann, L., Perrin, N., Rousset, F., 2006. Population demography and the evolution of helping behaviors. Evolution 60, 1137-1151.

Lehmann, L., Rousset, F., Roze, D., Keller, L., 2007. Strong reciprocity or strong ferocity? A population genetic view of the evolution of altruistic punishment The American Naturalist 170, 21-36.

Lions, S., van Baalen, M., 2008. Self-structuring in spatial evolutionary ecology Ecology Letters 11, 277-295.

Lumsden, C.J., Wilson, E.O., 1980. Translation of epigenetic rules of individual behavior into ethnographic patterns. Proceedings of the National Academy of Sciences of the United States of America 77, 4382-4386.

Lumsden, C.J., Wilson, E.O., 1981. Genes, Mind and Culture. Harvard University Press, MA.

Nakahashi, W., 2007. The evolution of conformist transmission in social learning when the environment changes periodically. Theoretical Population Biology 72 52-66.

Ohtsuki, H., Hauert, C., Lieberman, E., Nowak, M.A., 2006. A simple rule for the evolution of cooperation on graphs and social networks. Nature 441, 502-505.

Price, G.R., 1970. Selection and covariance. Nature 227, 520-521.

Richerson, P.J., Boyd, R., 2005. Not by Genes Alone. University of Chicago Press, Chicago.

Rogers, A.R., 1990. Group selection by selective emigration: The effects of migration and kin structure. American Naturalist 135, 398-413.

Rogers, E.M., 1995. Diffusion of innovations, 4th edn. Free Press, New York.

Rousset, F., 2004. Genetic Structure and Selection in Subdivided Populations. Princeton University Press, Princeton, NJ.

Rousset, F., Roze, D., 2007. Constraints on the origin and maintenance of genetic kin recognition. Evolution 61, 2320-2330.

Taylor, P.D., 1992. Altruism in viscous populations - an inclusive fitness model. Evolutionary Ecology 6, 352-356.

Taylor, P.D., Irwin, A.J., 2000. Overlapping generations can promote altruistic behavior. Evolution 54, 1135-1141.

Uyenoyama, M., Feldman, M.W., 1980. Theories of kin and group selection - a population-genetics perspective. Theoretical Population Biology 17, 380-414.

van Baalen, M., Rand, A., 1998. The unit of selection in viscous populations and the evolution of altruism. Journal of Theoretical Biology 193, 631-648.

Wakano, J.Y., Aoki, K., 2006. A mixed strategy model for the emergence and intensification of social learning in a periodically changing natural environment. Theoretical Population Biology 70, 486-497.

Wakano, J.Y., Aoki, K., Feldman, M.W., 2004. Evolution of social learning: A mathematical analysis. Theoretical Population Biology 66, 249-258.

Wolfram, S., 2003. Mathematica, 5th edn. Cambridge University Press, Cambridge. 